\title{
EQUIPAMIENTOS EDUCATIUOS DEL ÁREA URBANA DEFICITARIA CRITICA "GOLF CLUB". UNA APROXIMACIÓN AL ABORDAJE CUALI-CUANTITATIUO DE LA FRAGMENTACIÓN Y SEGREGACIÓN URBANA EN EL GRAN RESISTENCIA
}

Evelyn R. ABILDGAARD

eveabild@hotmail.com

Arquitecta. Integrante de los proyectos de investigación PI 12 SC01 SGCYT-UNNE y PICT 2014/0999 ANPCYT-FONCYT, dirigidos por el Dr. Arq Miguel Barreto. IIDVi, FAU-UNNE. Auxiliar Docente de la Cátedra Desarrollo Urbano I - FAU - UNNE

Palabras Clave: Educación; localización; integración social. Keywords: education; location; social integration

\section{RESUMEN}

Este artículo presenta avances de resultados de la investigación "Equipamientos colectivos para la integración social en Áreas Urbanas Deficitarias Críticas del Área Metropolitana del Gran Resistencia", realizada en el marco de la adscripción al Instituto de Investigación y Desarrollo en Vivienda (IIDVi). En esta primera instancia se cuantificaron y localizaron establecimientos educativos existentes en un área del Gran Resistencia denominada "AUDC Golf Club" y se realizaron entrevistas a familias que allí habitan para conocer sus experiencias y vivencias relacionadas con estos equipamientos. La propuesta metodológica utilizada busca poner en diálogo el abordaje tanto cuantitativo como cualitativo de la segregación espacial.

\begin{abstract}
This paper presents advances of a research called ("Equipamientos colectivos para la integración social en Áreas Urbanas Deficitarias Críticas del Área Metropolitana del Gran Resistencia", held within the framework of a Research Project of the Instituto de Investigación y Desarrollo en Vivienda (IIDVi). In this first instance were quantified and were located educational establishments existing in an area of Great Resistance called "AUDC Golf Club". Also were made interviews with families who live there to know about their experiences related to these establishments. The proposed methodology seeks to articulate both quantitative and qualitative approach of spatial segregation.
\end{abstract}




\section{OBJETIVOS}

Profundizar el marco teórico relativo al abordaje cuali-cuantitativo de la fragmentación y la segregación urbana; localizar y cuantificar equipamientos educativos del área urbana deficitaria crítica "Golf Club"; entrevistar a familias de distintos niveles de ingresos económicos que habitan en la AUDC, con la finalidad de detectar problemas de integración social.

\section{INTRODUCCIÓN}

La segregación socioespacial y la deficiente provisión de equipamientos colectivos en áreas urbanas consideradas como deficitarias críticas dentro del Gran Resistencia plantean (entre otros) interrogantes relacionados con la cantidad y calidad de infraestructura educativa existente en estas áreas y su capacidad para generar integración social.

Los resultados que se exponen en este artículo forman parte de un trabajo de investigación ${ }^{1}$ que estudia equipamientos colectivos y su capacidad para promover la integración social. Para ello, en una primera instancia se cuantificaron y localizaron los establecimientos educativos existentes en un área del Gran Resistencia denominada "AUDC Golf Club" y se realizaron entrevistas a familias que allí habitan para conocer sus experiencias y vivencias relacionadas con estos equipamientos. La etapa siguiente de la investigación consiste en el análisis espacial de estos equipamientos utilizando Sistemas de Información Geográfica (SIG) y entrevistas en establecimientos educativos del AUDC.

1 Realizado dentro del marco general del PI 12SC01 "Cuantificación y tipificación de áreas urbanas deficitarias críticas para la intervención integral del hábitat social del Gran Resistencia", dirigido por el Dr. Arq. MIGUEL BARRETO; contribuye además al PI 12C007 "Hábitat y Desigualdad Social. Antropología de las áreas urbanas deficitarias críticas en el Área Metropolitana del Gran Resistencia" dirigido por la Mg. Arq. ANDREA BENITTEZ. Ambos proyectos acreditados por la SGCYT-UNNE.
Se utilizó la metodología de una investigación cuanti-cualitativa, que tiene como sustento de concepción y abordaje del problema la teoría de la complejidad, según los planteos de autores como EDGARD MORIN (1990) y ROLANDO GARCÍA (2006), y la teoría de la integralidad del problema habitacional, según lo han desarrollado BARRETO y el equipo de investigación (BARRETO, 2010). Para abordar el tema de la segregación espacial se siguió la propuesta teórico-metodológica de GABRIELA MERA (2014).

El trabajo se inició con la profundización del marco teórico y la elaboración de los instrumentos de relevamiento y análisis de la información primaria; se recopiló y analizó información disponible en organismos públicos, documentos y fuentes estadísticas; se relevaron equipamientos educativos de la AUDC Golf Club, definida como caso de estudio; se realizaron entrevistas en tres unidades espaciales de dicha área (Parque Golf Club, Mujeres Argentinas y 24 de Diciembre) representativas de población de distintos niveles de ingreso económico.

\section{Educación y Segregación Social}

El sistema de equipamientos colectivos se define como "el conjunto de espacios y construcciones de uso público o privado, cuya función es la prestación de servicios para atender y satisfacer las necesidades recreativas, culturales, de salud, de educación, de abastecimiento, etc. requeridas por una comunidad, constituyéndose en componentes fundamentales de la estructuración, jerarquización y organización del tejido urbano" (MAYORGA HENAO, 2010: 38).

KAZTMAN (2001: 177) se refiere al sistema educativo como "el principal ámbito institucional que tiene la potencialidad de actuar como un crisol integrador, según sea su capacidad para 
"Cuando existe coincidencia entre la realidad y el imaginario de las personas y se responde a las necesidades expresadas, seguramente el espacio será 'apropiado' por la sociedad que lo habita."

generar contextos en que niños y adolescentes pobres tengan la posibilidad de mantener una relación cotidiana con sus pares de otros estratos y desarrollar con ellos códigos comunes y vínculos de solidaridad y afecto bajo condiciones de igualdad". Afirma además que "una política educativa, que parta por distribuir adecuadamente este tipo de equipamientos, puede hacer una importante contribución a la equidad en la distribución de activos de capital social, al facilitar la construcción de redes de estudiantes de composición social heterogénea".(KAZTMAN, Óp. Cit.: 174).

Al referirse a los espacios para la integración social, SALAZAR Y DEVOTO MAGOFKE (2010: 198) expresan que es posible caracterizar cada sector urbano según su escala, su vinculación con el resto de la ciudad, su diseño y materialidad, o también por los beneficios que otorga a la convivencia de sus habitantes. Cuando existe coincidencia entre la realidad y el imaginario de las personas y se responde a las necesidades expresadas, seguramente el espacio será "apropiado" por la sociedad que lo habita. Y es la apropiación la que verifica el vínculo habitante - territorio.

Para la CEPAL/CELADE (2002), "el espacio residencial no es el único donde interactúan los individuos, y que la eventual falta de contacto vecinal puede contrarrestarse por la existencia de otros ámbitos de interacción". KAZTMAN (Óp. Cit.: 173) señala que cuando los ingresos de la población se alejan del promedio, los hogares favorecidos aumentan su propensión a adquirir servicios de mejor calidad. Cuando esta conducta se extiende a la prestación de servicios básicos, como la educación, se producen cambios importantes en la estructura social, los cuales acentúan los mecanismos de aislamiento social.

MERA (2014) recopila conceptos de autores que "han buscado tomar distancia de la tendencia a concebir a la segregación espacial reducida a lo físico-residencial, para incorporar aspectos del habitar, como prácticas, representaciones y formas de apropiación del espacio urbano". A continuación se exponen los autores citados por MERA que explicitan el posicionamiento teórico que servirá de marco para analizar el caso de estudio.

WHITE (1983) propone distinguir entre la segregación geográfica, que remite a la clásica definición de la segregación como una distribución desigual en un espacio físico, y la segregación sociológica, que alude a la ausencia de interacción y contacto entre los grupos, incorporándose de esta manera una mirada crítica sobre la acepción clásica de esta noción, considerando que puede haber discrepancias y tensiones entre distancias físicas y distancias sociales, y que la proximidad geográfica no necesariamente involucra interacción (ni la distancia física implica aislamiento). Lo mismo opinan BOND Y PARKER (2000) al expresar que "la cercanía geográfica no garantiza el intercambio ni asegura afinidad o armonía entre los grupos".

En el contexto latinoamericano, entre los autores que han buscado complejizar la noción de segregación espacial, SABATINI propone considerar la existencia no solo de una dimensión objetiva de la segregación, que se vincula con la tendencia de un grupo a concentrarse en algunas zonas de la ciudad y la conformación de áreas socialmente homogéneas; sino que incorpora también una dimensión subjetiva, que refiere a las percepciones, al prestigio (o desprestigio) social de las distintas áreas; es decir, a "las imágenes, reputación y estigmas territoriales asignados por la población de la ciudad a algunos de sus vecindarios" (SABATINI, 2003: 7).

CAPRÓN Y GONZÁLEZ ARELLANO (2006) señalan otros dos sentidos desde los que puede entenderse la segregación espacial: como acceso desigual a 
los servicios y equipamientos urbanos, es decir, como un fenómeno que remite a la movilidad y, por otro lado, como una forma de espacialización de la distancia social entre los grupos, en particular en términos de ajustes y conflictos sociales.

Para finalizar esta recopilación de conceptos, se incluye el aporte que brinda desde la antropología RAMIRO SEGURA (2010, 2012), quien propone incorporar en los estudios de la segregación residencial dos dimensiones esenciales: por un lado, el territorio barrial (cómo lo simbolizan los propios habitantes) y, por el otro, la territorialidad de las prácticas de los actores, lo que implica reconstruir sus redes de relaciones, dentro y fuera del espacio en cuestión.

Esta perspectiva le permite discutir con "la idea generalizada de una total separación, aislamiento y exclusión de los espacios segregados, marginados y estigmatizados", para ver, en cambio, que si bien se producen procesos que empujan al aislamiento a los grupos que viven "tras las fronteras", constantemente se tejen estrategias que establecen puentes y pasajes entre tales ámbitos separados.

Sobre la base de estos conceptos teóricos, MERA (Óp. Cit.) propone analizar el fenómeno multidimensional de la segregación espacial desde una metodología que combine:

- Un abordaje macroanalítico y cuantitativo, que permita dar cuenta de la existencia de formas de desigualdad o concentración espacial en los patrones de asentamiento de los grupos a nivel intraurbano.

- Un abordaje microsocial y cualitativo centrado en la experiencia e interacción social, en el marco de la cual se construyen fronteras simbólicas que condicionan las dinámicas socioespaciales. (Fig.1).

\section{DESARROLLO}

\section{El Área Urbana Deficitaria Crítica Golf Club}

Un Área Urbana Deficitaria Crítica (AUDC) se define como una "porción de territorio periurbano

Figura 1. Propuesta teórico-metodológica para abordar la segregación espacial

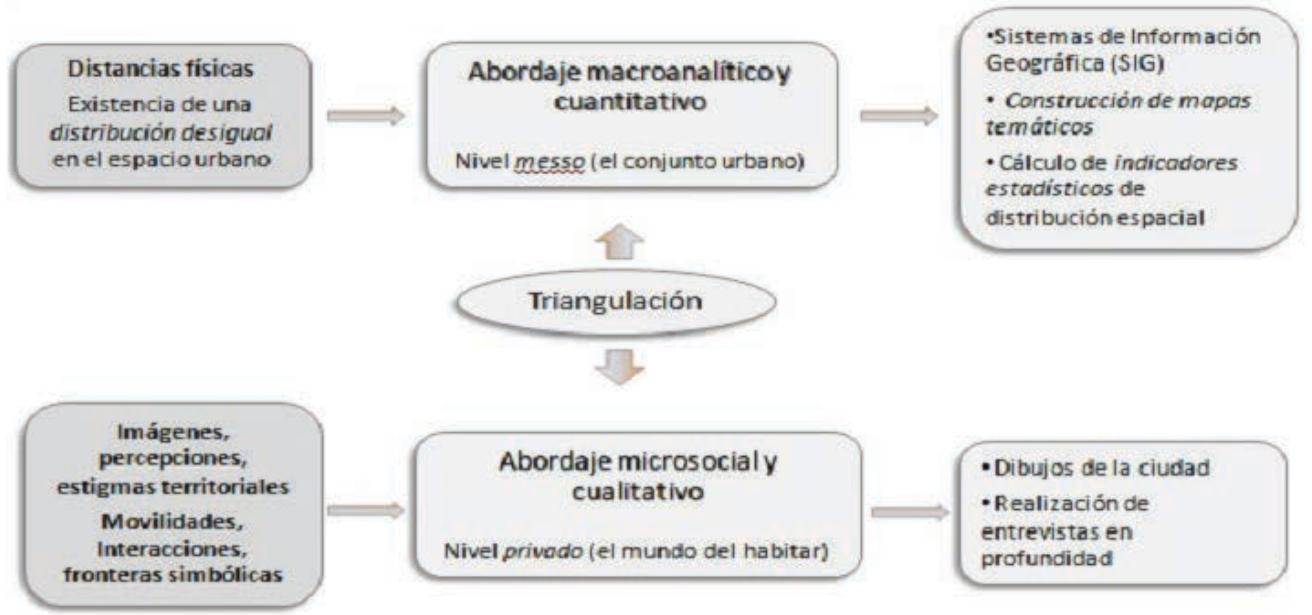

Fuente: MERA, GABRIELA (2014) 
Figura 2. Unidades espaciales (barrios) AUDC Golf Club

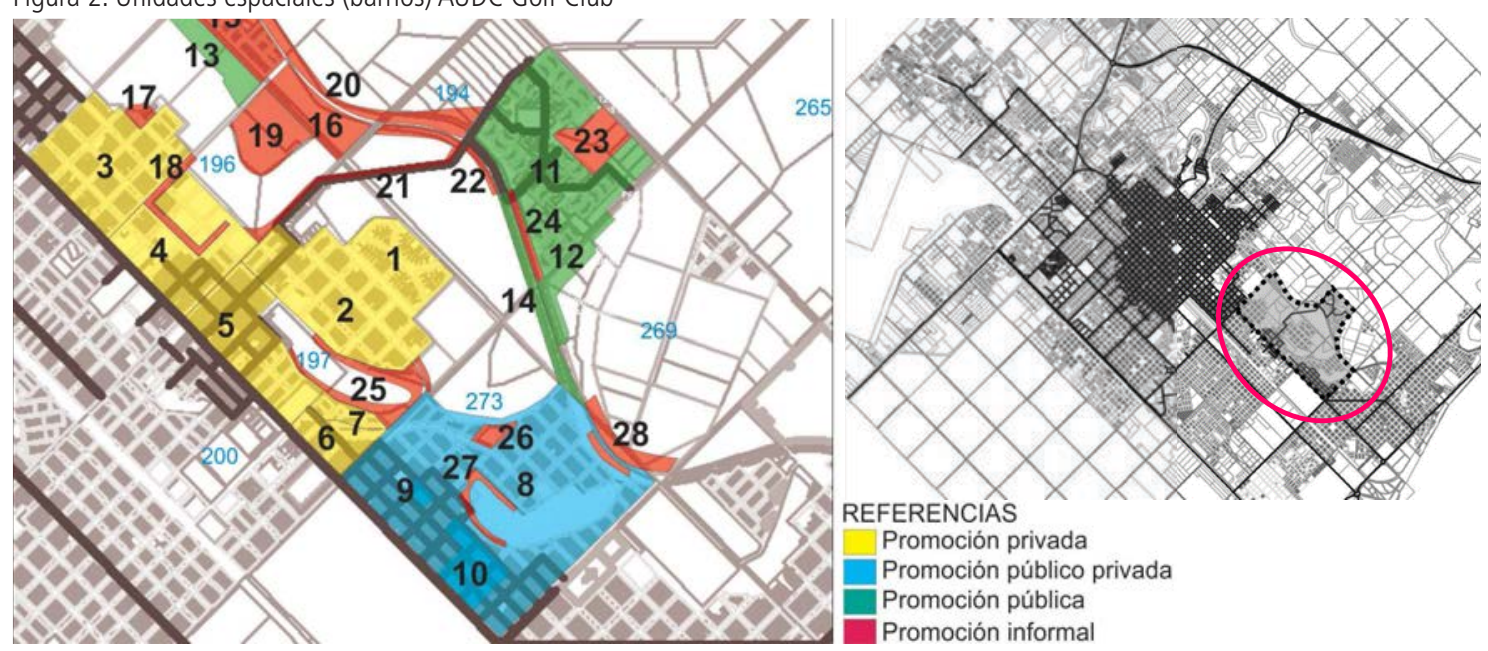

Fuente: PI-UNNE 12SC01. Cuantificación y tipificación de Áreas Urbanas Deficitarias Críticas para la intervención integral del hábitat social del Gran Resistencia

en el que conviven unidades espaciales (barrios) internamente homogéneos o cohesionados, pero muy desiguales entre ellos, que se distingue por ser un territorio desarticulado (...) producto de las particularidades de la segregación espacial del proceso de urbanización de las últimas décadas, que ha generado un espacio residencial altamente fragmentado" (BARRETO ET ÁL., 2014).

Se toma como caso de estudio el AUDC denominada por el PI12SC01 como "Golf Club", compuesta por veintiocho unidades espaciales (barrios y asentamientos), según lo graficado en la figura 2 .

Estimaciones realizadas en el año 2013 por el equipo de investigación, utilizando el método indirecto², contabilizaron 5781 viviendas, con aproximadamente 20.812 habitantes.
Estas veintiocho Unidades Espaciales (UE) fueron clasificadas según los agentes promotores que les dieron origen y el nivel de ingresos económicos relativos de sus habitantes y expuestas en un cuadro, del cual, para este trabajo, se extrajo la información referida a las tres unidades espaciales seleccionadas para realizar las entrevistas (cuadro 1).

\section{Abordaje cuantitativo. Distribución espacial de los establecimientos educativos}

Para la localización y posterior cuantificación de los equipamientos educativos de AUDC Golf Club se utilizaron los datos SIG contenidos en las tablas del shapefile correspondiente al total de escuelas de nivel inicial, primario y medio de la provincia de Chaco realizándose selecciones por atributos:

Cuadro 1. Unidades espaciales AUDC Golf Club

\begin{tabular}{|l|l|c|c|c|c|}
\hline \multicolumn{2}{|c|}{$\begin{array}{c}\text { Unidades espaciales } \\
\text { (Barrios) }\end{array}$} & Promoción & $\begin{array}{c}\text { Cantidad de } \\
\text { viviendas }\end{array}$ & $\begin{array}{c}\text { Cantidad de } \\
\text { habitantes }\end{array}$ & $\begin{array}{c}\text { Nivel relativo de } \\
\text { ingresos } \\
\text { económicos }\end{array}$ \\
\hline 1 & Parque Golf Club & Privada & 47 & 169 & Alto \\
\hline 11 & Mujeres argentinas & Pública & 1.030 & 3.708 & Medio y Medio bajo \\
\hline 16 & 24 de Diciembre & Informal & 100 & 360 & Bajo \\
\hline & TOTAL & & $\mathbf{5 . 7 8 1}$ & $\mathbf{2 0 . 8 1 2}$ & \multicolumn{1}{|l}{} \\
\cline { 1 - 3 } & & &
\end{tabular}

Fuente: PI-UNNE 12SC01. Cuantificación y tipificación de Áreas Urbanas Deficitarias Críticas para la intervención integral del hábitat social del Gran Resistencia

2 El método indirecto se utiliza para suplir la falta de información censal desagregada del año 2010. Consiste en el conteo de viviendas a partir de fotos satelitales y la estimación de población que la habita sobre la base del promedio de personas por hogar que arroja el censo. Ha sido utilizado en otras investigaciones (BARRETO, ALCALÁ, BENÍTEZ, DEPETTRIS Y KISZKA, 2012) y por otros investigadores (LIVERT AQUINO, 2006) y organismos de otros países (MINISTERIO DAS CIDADES, 2010). la primera para conocer los establecimientos educativos del AMGR y luego para saber los del AUDC Golf Club. El mapa base corresponde al shapefile "Radios y Fracciones" del Censo Nacional de Población Hogares y Viviendas (INDEC, 2010), ya que en la segunda instancia de esta investigación se utilizarán indicadores estadísticos. 


\section{Localización de equipamientos educativos}

La figura 3 representa la localización de los establecimientos educativos de nivel inicial, primario y medio tanto a nivel provincial como a nivel metropolitano.

El cuadro 2 cuantifica las escuelas de nivel inicial, primario y medio del AMGR. Del total de 332 escuelas, el $77.41 \%$ se encuentra en Resistencia, el $11.45 \%$ en Barranqueras, el 6.32 $\%$ en Fontana y en Puerto Vilelas el $4.82 \%$. De las 127 escuelas de nivel inicial, el $79.53 \%$ está en Resistencia, el $10.24 \%$ en Barranqueras, el $5.51 \%$ en Fontana y el $4.72 \%$ en Puerto Vilelas. Las 121 escuelas de nivel primario representan los siguientes porcentajes: Resistencia $74.38 \%$ Barranqueras $11.57 \%$. Fontana $7.44 \%$ y Puerto

Figura 3. Localización de Equipamientos Educativos en la Provincia de Chaco y en el AMGR

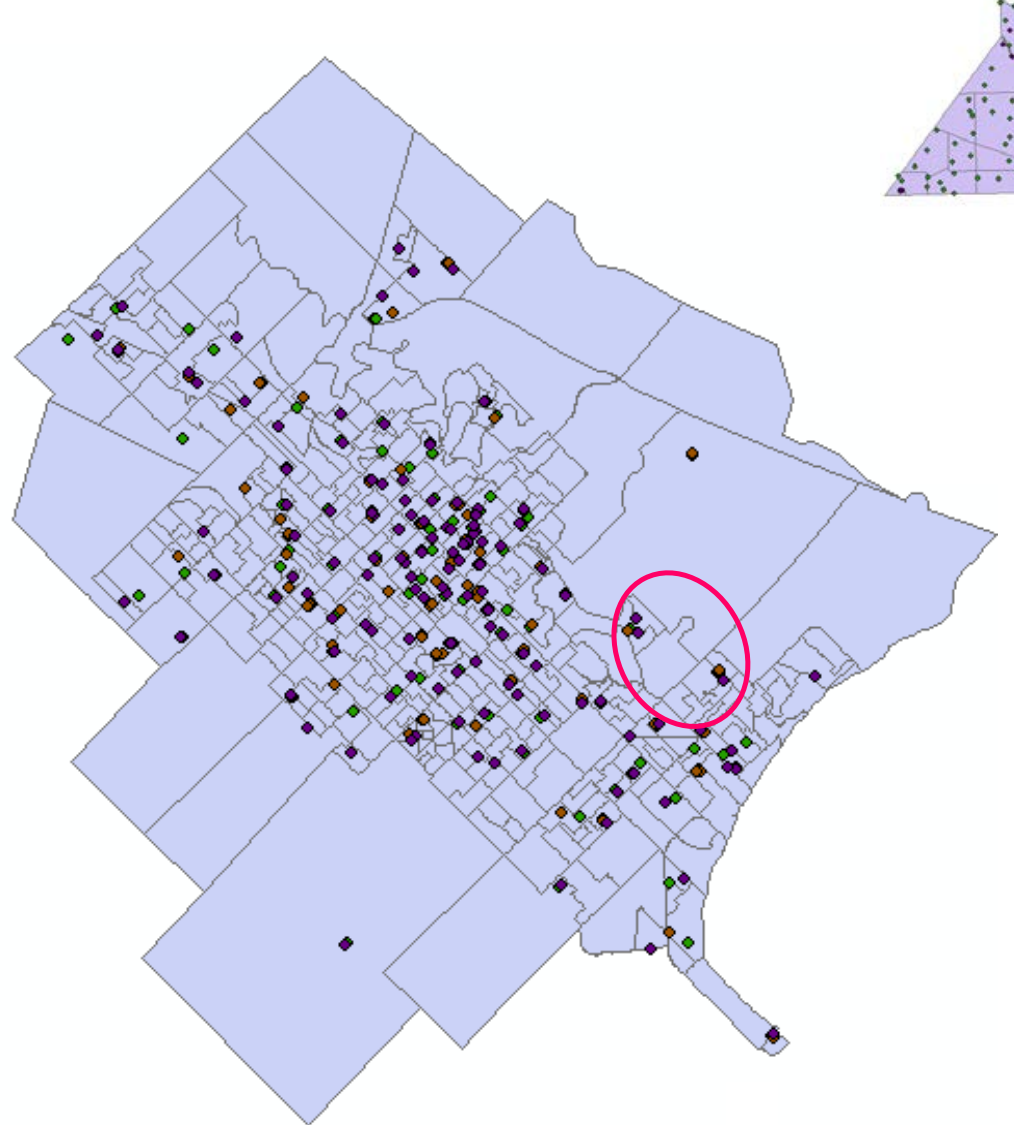

Referencias:

Nivel Inicial

Nivel Primario

Nivel Secundario

AUDC Golf Club

AMGR

Fuente: elaboración propia sobre la base de datos del Ministerio de Educación y del INDEC 
Cuadro 2. Escuelas en el AMGR

\begin{tabular}{|l|c|c|c|c|c|}
\hline AMGR & Resistencia & Barranqueras & Fontana & Puerto Vilelas & Total \\
\hline Nivel Inicial & 101 & $\mathbf{1 3}$ & $\mathbf{7}$ & $\mathbf{6}$ & 127 \\
\hline Nivel Primario & $\mathbf{9 0}$ & $\mathbf{1 4}$ & $\mathbf{9}$ & $\mathbf{8}$ & 121 \\
\hline Nivel Medio & $\mathbf{6 6}$ & $\mathbf{1 1}$ & $\mathbf{5}$ & $\mathbf{2}$ & 84 \\
\hline Total & 257 & 38 & 21 & 16 & 332 \\
\hline
\end{tabular}

Elaboración propia sobre la base de datos del Ministerio de Educación, Ciencia y Tecnología de la provincia del Chaco

Vilelas $6.61 \%$. Las 84 de nivel medio: Resistencia $78.57 \%$, Barranqueras $13.10 \%$, Fontana 5.95 $\%$ y Puerto Vilelas $2.38 \%$.

En el cuadro 3 se muestra la cantidad de escuelas urbanas y rurales de los cuatro municipios que integran el AMGR. Resistencia, al ser el municipio de mayor concentración urbana, solamente tiene cinco escuelas rurales. Fontana y Barranqueras no registran escuelas rurales. El caso inverso a Resistencia se da en Puerto Vilelas, donde el número de escuelas de nivel inicial y primario del área rural supera a las urbanas.

Cuadro 3. Escuelas por municipios del AMGR

\begin{tabular}{|l|c|c|}
\hline \multicolumn{1}{|c|}{ RESISTENCIA } & Urbana & Rural \\
\hline Nivel Inicial & $\mathbf{9 9}$ & $\mathbf{2}$ \\
\hline Nivel Primario & $\mathbf{8 8}$ & $\mathbf{2}$ \\
\hline Nivel Medio & 65 & $\mathbf{1}$ \\
\hline Total & 252 & 5 \\
\hline
\end{tabular}

\begin{tabular}{|l|c|c|}
\hline \multicolumn{1}{|c|}{ FONTANA } & Urbana & Rural \\
\hline Nivel Inicial & $\mathbf{7}$ & - \\
\hline Nivel Primario & 9 & - \\
\hline Nivel Medio & $\mathbf{5}$ & - \\
\hline Total & 21 & - \\
\hline
\end{tabular}

Para conocer el tipo de gestión de las escuelas de Resistencia, se realizó una nueva selección por atributos como primera aproximación a las del AUDC Golf Club. El cuadro 4 indica que predominan las escuelas públicas de gestión estatal. La EPGSI N. ${ }^{\circ} 1$ (EX-UEP N. ${ }^{\circ}$ 30) es una Escuela de Gestión Social Indígena (a).

Para identificar las escuelas del AUDC Golf Club se recurrió a superponer la capa correspondiente a los equipamientos educativos sobre una imagen aérea georreferenciada (orto-fotografía del Instituto Geográfico Nacional) para tener, como

Elaboración propia sobre la base de datos del Ministerio de Educación, Ciencia y Tecnología de la provincia del Chaco

Cuadro 4. Escuelas en Resistencia (según tipo de gestión)

\begin{tabular}{|l|c|c|c|c|}
\hline \multicolumn{1}{|c|}{ RESISTENCIA } & Estatal & Privada & $\begin{array}{c}\text { Gestión Social } \\
\text { Cooperativa }\end{array}$ & Total \\
\hline Nivel Inicial & $\mathbf{6 8}$ & $\mathbf{3 2}$ & $\mathbf{1}$ (a) & 101 \\
\hline Nivel Primario & $\mathbf{6 7}$ & $\mathbf{2 3}$ & - & 90 \\
\hline Nivel Medio & $\mathbf{4 5}$ & $\mathbf{2 0}$ & $\mathbf{1}(\mathbf{a})$ & 66 \\
\hline Total & 180 & 75 & 2 & 257 \\
\hline
\end{tabular}

Elaboración propia sobre la base de datos del Ministerio de Educación, Ciencia y Tecnología de la provincia del Chaco 
define MERA, "una vista desde arriba". Luego se realizaron relevamientos físicos para corroborar los datos del cuadro 5.

La Liguria, Mujeres Argentinas, Don Bosco y Villa Los Lirios son solamente cuatro de las veintiocho unidades espaciales (barrios) que conforman el área urbana deficitaria crítica Golf Club que cuentan con establecimientos escolares. Las quince escuelas del área representan el 5.84 $\%$ del total de escuelas de Resistencia. La población estimada de la AUDC Golf Club (20.812 habitantes) representa el $7.20 \%$ de la población del municipio de Resistencia (290.723 habitantes). Los cuadros 6, 7, 8 y 9 detallan las escuelas de nivel inicial, primario y medio que se encuentran en cada UE del AUDC. Todas son escuelas públicas de gestión estatal.

En La Liguria existen dos establecimientos educativos de nivel inicial, dos primarios (en uno de ellos funciona una escuela de adultos) y uno de nivel medio. En Mujeres Argentinas existen dos establecimientos educativos de nivel inicial, dos primarios y dos de nivel medio. En el año 2013 dos cursos $\left(4 .^{\circ}\right.$ y $\left.5 .^{\circ}\right)$ de la CEP N..$^{\circ} 25$ corrieron el riesgo de ser cerrados debido al escaso cupo. Manifestaciones de padres y alumnos impidieron que se produzca el cierre.

Cuadro 5. Escuelas en el AUDC Golf Club

\begin{tabular}{|l|c|c|c|c|c|}
\hline AUDC GC & La Liguria & $\begin{array}{c}\text { Mujeres } \\
\text { Argentinas }\end{array}$ & Don Bosco & $\begin{array}{c}\text { Villa Los } \\
\text { Lirios }\end{array}$ & Total \\
\hline Nivel Inicial & $\mathbf{2}$ & $\mathbf{2}$ & $\mathbf{1}$ & - & 5 \\
\hline Nivel Primario & $\mathbf{2}$ & $\mathbf{2}$ & $\mathbf{1}$ & $\mathbf{1}$ & 6 \\
\hline Nivel Medio & $\mathbf{1}$ & $\mathbf{2}$ & $\mathbf{1}$ & - & 4 \\
\hline Total & 5 & 6 & 3 & 1 & 15 \\
\hline
\end{tabular}

Elaboración propia sobre la base de datos del Ministerio de Educación, Ciencia y Tecnología de la provincia del Chaco

Cuadro 6. Escuelas en la UE La Liguria

\begin{tabular}{|c|c|c|c|c|}
\hline $\mathrm{N}^{\circ}$ en Mapa & Nombre & Nivel & Dirección & Gestión \\
\hline 1.1 & $\begin{array}{l}\text { EGB N }{ }^{\circ} 13 \text { Ministro Benjamin } \\
\text { Victorica. }\end{array}$ & Primario & Av. 9 de Julio 3160 & $\begin{array}{c}\text { Educación Pública } \\
\text { Estatal }\end{array}$ \\
\hline 1.2 & $\begin{array}{l}\text { CEP N }{ }^{\circ} 54 \text { (Ex-ENS } 57 \text { Pablo } \\
\text { Ricchieri) }\end{array}$ & Medio & Av. 9 de Julio 3140 & $\begin{array}{c}\text { Educación Pública } \\
\text { Estatal }\end{array}$ \\
\hline 1.3 & $\begin{array}{l}\text { Jardín de Infantes } \mathrm{N}^{\circ} 46 \\
\text { Baldomero Fernández Moreno. }\end{array}$ & Inicial & Hornero $\mathrm{N}^{\circ} 120$ & $\begin{array}{c}\text { Educación Pública } \\
\text { Estatal }\end{array}$ \\
\hline 1.4 & EGB N ${ }^{\circ} 319$ Juan Manuel Rossi. & Primario (*) & $\begin{array}{l}\text { Aristóbulo del Valle } \\
\qquad N^{\circ} 195\end{array}$ & $\begin{array}{c}\text { Educación Pública } \\
\text { Estatal }\end{array}$ \\
\hline 1.5 & $\begin{array}{l}\text { J. de Infantes y J. Maternal } \\
N^{\circ} 43 \text {. María Luisa Roques }\end{array}$ & Inicial & Av 9 de julio $N^{\circ} 3170$ & $\begin{array}{c}\text { Educación Pública } \\
\text { Estatal }\end{array}$ \\
\hline
\end{tabular}

$\left(^{*}\right)$ Escuela Primaria y de Adultos

Elaboración propia sobre la base de datos del Ministerio de Educación, Ciencia y Tecnología de la provincia del Chaco 
Cuadro 7. Escuelas en la UE Mujeres Argentinas

\begin{tabular}{|c|c|c|c|c|}
\hline $\mathrm{N}^{\circ}$ en Mapa & Nombre & Nivel & Dirección & Gestión \\
\hline 2.1 & $\begin{array}{l}\text { CEP N²5 Cacica Mercedes } \\
\text { Dominga (Ex - ENS } 81 \text { Golf Club) }\end{array}$ & Medio & $\begin{array}{l}\text { Mz. } 43 \text { - Pc. } 51 \text { - Barrio } \\
\text { Mujeres Argentina }\end{array}$ & $\begin{array}{c}\text { Educación Pública } \\
\text { Estatal }\end{array}$ \\
\hline 2.2 & $\begin{array}{l}\text { EEP N }{ }^{\circ} 108 \text { Inés Manuela García } \\
\text { de Márquez }\end{array}$ & Primario y Medio & Alfonsina Storni 145 & $\begin{array}{c}\text { Educación Pública } \\
\text { Estatal }\end{array}$ \\
\hline 2.3 & $\begin{array}{l}\text { Escuela Primaria para Adultos } \\
N^{\circ} 30 \text { Juan XXIII }\end{array}$ & Primario & $\begin{array}{l}\text { Aristóbulo del Valle } \\
195\end{array}$ & $\begin{array}{c}\text { Educación Pública } \\
\text { Estatal. }\end{array}$ \\
\hline 2.4 & $\begin{array}{l}\text { Jardin de Infantes } \mathrm{N}^{\circ} 112 \text { - Olga } \\
\text { Cossettini. }\end{array}$ & Inicial & $\begin{array}{l}\text { Mujeres Argentinas } \\
\text { Pc. } 116\end{array}$ & $\begin{array}{c}\text { Educación Pública } \\
\text { Estatal }\end{array}$ \\
\hline 2.5 & $\begin{array}{l}\text { Jardin de Infantes } N^{0} 112 \text { - Olga } \\
\text { Cossettini. }\end{array}$ & Inicial & Mujeres Argentinas & $\begin{array}{c}\text { Educación Pública } \\
\text { Estatal }\end{array}$ \\
\hline
\end{tabular}

Elaboración propia sobre la base de datos del Ministerio de Educación, Ciencia y Tecnología de la provincia del Chaco

Cuadro 8. Escuelas en la UE Don Bosco

\begin{tabular}{|c|l|l|l|l|}
\hline$N^{\circ}$ en Mapa & Nombre & Nivel & Dirección & Gestión \\
\hline 3.1 & Jardín de Infantes N $N^{\circ} 137$ & Inicial & Inspector Patiño S/N & $\begin{array}{c}\text { Educación Pública } \\
\text { Estatal }\end{array}$ \\
\hline 3.2 & EGB N 893 & Primario y Medio & $\begin{array}{l}\text { Sargento Cabral } \\
1801\end{array}$ & $\begin{array}{c}\text { Educación Pública } \\
\text { Estatal }\end{array}$ \\
\hline
\end{tabular}

Elaboración propia sobre la base de datos del Ministerio de Educación, Ciencia y Tecnología de la provincia de Chaco

Cuadro 9. Escuelas en la UE villa Los Lirios

\begin{tabular}{|c|l|l|l|l|}
\hline$N^{\circ}$ en Mapa & Nombre & Nivel & Dirección & Gestión \\
\hline 4.1 & $\begin{array}{l}\text { EGB No } 577 \text { del } \\
\text { Sesquicentenario }\end{array}$ & Primario & $\begin{array}{l}\text { Brown 1721. Villa Los } \\
\text { Lirios }\end{array}$ & $\begin{array}{c}\text { Educación Pública } \\
\text { Estatal }\end{array}$ \\
\hline
\end{tabular}

Elaboración propia sobre la base de datos del Ministerio de Educación, Ciencia y Tecnología de la provincia del Chaco

En el barrio Don Bosco funciona un establecimiento educativo de nivel inicial, uno de nivel primario y uno de nivel medio (cuadro 8). En villa Los Lirios funciona únicamente un establecimiento educativo de nivel primario (cuadro 9).

\section{Abordaje cualitativo. Relaciones e interacciones sociales}

Para conocer las prácticas y las representaciones subjetivas de los habitantes de la AUDC Golf Club se realizaron entrevistas en tres unidades espaciales a familias de distintos ingresos económicos: Parque Golf Club, de ingresos altos; Mujeres argentinas de ingresos medios y medio-bajos: 24 de diciembre, de bajos ingresos.

\section{Entrevistas $^{3}$}

El análisis ${ }^{4}$ de las entrevistas se realizó a partir de las estrategias de localización residencial de las familias y sus expectativas de poder lograr una trayectoria ascendente en la escala social a través de la educación. La educación, analizada en términos

3. Entrevistas realizadas por ABILDGAARD, E., CAZORLA V., LEDESMA, E. Y PUNTEL, L. dentro del marco general del PI 12 SC01 "Cuantificación y tipificación de áreas urbanas deficitarias críticas para la intervención integral del hábitat social del Gran Resistencia". IIDVi, FAU-UNNE.

4. Los casos analizados forman parte del trabajo denominado "Desigualdad y fragmentación durante una década contradictoria. Trayectorias sociales y localizaciones residenciales posneoliberales en una Área Urbana Deficitaria Crítica de Resistencia (Argentina)". Autores: BARRETO, M.; BENITEZ, A.; ABILDGAARD, E.; CAZORLA, V. y PUNTEL, L. de lo que BOURDIEU (2001) denomina capital cultural institucionalizado, es decir, expresada en la obtención de títulos de diferentes grados, ha sido siempre percibida como uno de los recursos más válidos para realizar una trayectoria social ascendente y mejorar la posición social.

Mercedes (48 años), vecina del barrio Parque Golf Club, posee alto capital cultural incorporado e institucionalizado a través de la obtención de estudios universitarios hasta el nivel de posgrado. En su infancia y adolescencia vivió en La Liguria; en ese tiempo no existían allí establecimientos educativos primarios ni secundarios; por lo tanto, sus padres la enviaron a ella y a sus hermanas a escuelas del centro de la ciudad: "Las tres hermanas fuimos a una cooperativa educativa en la primaria. Mi madre era la directora. La escuela era bastante progre. En la dictadura fue cerrada por los militares. Entonces fuimos a la escuela $N .^{\circ}$ 319. Yo fui desde $4 .^{\circ}$ a 7. ${ }^{\circ}$ ahí. En el barrio no existía escuela secundaria; las tres fuimos al Colegio Nacional, que no tenía una orientación específica, sino un pantallazo general. El Nacional y la Normal eran en esa época las escuelas públicas donde iban las personas de nivel socioeconómico medio. Ahora la Normal bajó mucho su nivel. Y para entrar al Nacional los chicos se preparan un año antes con profesores particulares para rendir el examen de ingreso".

En el año 1997 Mercedes y su marido se mudaron al barrio Parque Golf Club. En este barrio de clase media-alta, no existen equipamientos educativos. 
Aunque por un breve período envió a sus hijas a una escuela privada (Instituto Amici), prefiere educarlas en establecimientos públicos. "Yo, por una cuestión ideológica, quería que mis hijas fueran a la escuela pública. La mayor fue al Jardín N. ${ }^{\circ} 28$, ahí, cerca de la plaza España. Y la primaria a la escuela $N{ }^{\circ} 33$, pero en un año cambiaron cinco veces de maestra, había paros... Después fuimos a vivir a Italia, para hacer la maestría. Alli también fueron a una escuela pública. Gabriela a 2. ${ }^{\circ}$ e Irina a jardín. Gabriela enseguida aprendió a hablar en italiano, pero la más chiquita estaba en el jardín con chicos rusos y de otros países... y le costó más. Después aprendió y hablaban entre ellas en italiano. Cuando volví las Ilevé al Amici. Irina entró a Jardín de 4 y Gabriela en dos meses terminó tercer grado. La escuela secundaria la hicieron en el Colegio Nacional. Ahí sí quise nuevamente que vayan a una escuela pública, para foguearse, para abrirse al mundo".

Víctor (50 años), vecino del barrio Mujeres Argentinas, considera que las relaciones que se establecen en las escuelas del barrio a partir de la incorporación de alumnos provenientes de los asentamientos aledaños causan desprestigio social, y opta por enviar a sus hijas a escuelas localizadas en otro barrio. "Venían los de las periferias, de allá... Entonces, ¿qué haces vos... cuando tenés un hijo que se empieza a juntar con este? Acá no hay alumnos... andá a fijarte en esa secundaria: no hay alumnos. ¿Por qué se fueron todos? Porque empiezan los malos hábitos, las malas cosas... el... la droga... todo un desastre. Yo acá viví ocho, nueve años, que nunca pasó... después de eso y cuando se empiezan a juntar de otros lados... entonces yo decidí mandarle a otro lado, donde la junta no sea esa; porque vos acá lo que tenés que una chica que esté acá o que esté allá, tenés tu horario que entran por ejemplo a la 7.30 am y a la 10.30 están libres, tenés diez personas en tus escaleras allá haciendo quilombo".
Estas percepciones son las que SABATINI (2003: 7) define como imágenes, reputación y estigmas territoriales asignados por la población de la ciudad a algunos de sus vecindarios.

Natalia (22 años), madre soltera, vive en el asentamiento 24 de diciembre. Diversas circunstancias en su vida hicieron que su trayectoria social haya sido descendente, tal como lo relata ella: "Yo fui a la 41, que está allá por la Pellegrini y Ayacucho, o sea, desde primer grado y hasta sexto, a mitad de año más o menos, pero después que falleció mi papá, mi mamá nos cambió de escuela porque ella no nos podía llevar. Antes nos llevaba mi papá nomás, después falleció él y nos cambió a los tres acá y ahí terminamos. A mí no me gustó nunca, no quería ir, no quería saber nada de esa escuela (...) Eran todos distintos a mí. Era como que ellos ya tenían todo lo que iban a hacer. Ellos por ejemplo, salieron y se fueron todos a la del Golf y yo no, yo fui a la 45, rendí el examen y entre ahí. Acá la escuela del Golf también es un desastre la secundaria".

En narraciones como la de Natalia queda claro que en la obtención de un título se depositan aspiraciones importantes para lograr trayectorias ascendentes tanto en su propia vida como en la de su hija. Sin embargo, en esta historia se evidencia que no es el capital institucionalizado (el título) por sí mismo el que puede ayudar en esa trayectoria, sino también que esto está muy ligado al tipo de institución en que se lo adquiere: "Ahora está yendo al jardín acá, pasando la 9 de Julio, a una cuadra más o menos, 123 creo que es el jardín. Y ahora ya termina el de 5 ya. A la primaria ahora yo le quise anotar cerca del hospital Perrando, en la 116 y ya no conseguí más bancos y hoy mandé a preguntar acá en el barrio nomás, pero no me simpatiza mucho acá en el barrio, pero por lo menos en primer grado, porque ya es difícil que consiga". 
"No basta solo con saber cuántas escuelas hay, dónde están localizadas y si son suficientes o insuficientes para el área de cobertura; es necesario también conocer las vivencias, experiencias y expectativas de quienes habitan el área en estudio."

Así, en las escuelas primarias como las que ella tiene cerca del barrio informal en el que ha tenido que ir a vivir por necesidad o en escuelas secundarias próximas, no se depositan muchas expectativas de mejoría; por el contrario, a través de su experiencia, considera que en escuelas de este tipo se adquirirán conductas nada favorables a la trayectoria social que aspira para ella y su hija. Los casos de Natalia y de Mercedes tienen en común que en sus respectivos barrios no existen establecimientos escolares. Sin embargo, la primera encuentra restringida su elección debido a que no cuenta con los recursos económicos suficientes. Tal como afirma GOTMAN (1990), "Es por medio de los recursos como las estrategias individuales y las condicionantes estructurales interactúan".

ANHEIER ET ÁL. (1995) señala que lo que distingue las posiciones de élite de las demás son diferencias significativas en el capital cultural y simbólico. En el caso de la vecina del Parque Golf Club es evidente que a través de la posibilidad de elección de una localización residencial en un barrio más exclusivo, que le permitió la disposición de su capital económico, ella buscó expresar y reforzar su búsqueda de una trayectoria social ascendente, ligada a la obtención de capitales culturales en distintas formas.

\section{CONCLUSIONES}

A través del "diálogo" entre el espacio social y el poder simbólico (BOURDIEU, 1996), es posible analizar la segregación urbana. No basta solo con saber cuántas escuelas hay, dónde están localizadas y si son suficientes o insuficientes para el área de cobertura; es necesario también conocer las vivencias, experiencias y expectativas de quienes habitan el área en estudio. Mediante las narrativas familiares se pudo conocer que determinadas localizaciones no contribuyen a trayectorias sociales favorables para mejorar la posición social, sino que llevan a que se reproduzcan las condiciones padecidas. Algunas instituciones educativas tienen un mejor reconocimiento que otras, no solo en la calidad de la educación y del título obtenido, sino también en el tipo de relaciones sociales que en ellas se realizan. $Y$ estas diferencias tienen una estrecha relación con las localizaciones de los establecimientos en los diferentes barrios y zonas de la ciudad.

La educación formal cumple un rol importante en la estructuración e integración de niños y adolescentes a la sociedad, en tanto medio que incide de manera preponderante en la adquisición de capitales sociales y culturales fundamentales para la inserción en el mundo laboral de los adultos. La incorporación de capacidades (saberes), la obtención de capitales institucionalizados (títulos) y las relaciones iniciales que se tejen con sus semejantes (capital social) inciden en las posibilidades de pertenencia e integración social futura. Sin embargo, no todas las instituciones educativas son iguales ni brindan las mismas posibilidades.

En el AUDC Golf Club, en los barrios donde viven sectores de bajos recursos, las instituciones educativas en muchos casos no favorecen este proceso y algunos padres por diferentes circunstancias tienen restringidas posibilidades de movilidad y por ende limitadas posibilidades de elección, debido a que no cuentan con los recursos suficientes para llevar a sus hijos a otras instituciones. En estos casos, la escuela, en lugar ser un medio que favorece la integración social, contribuye a que se reproduzcan las condiciones y la desigualdad social. 
ANHEIER, Helmut; GERHARDS, Jurgen y ROMO, Frank (1995) "Forms of Capital and Social Structure in Cultural Fields: Examining Bourdieu's Social Topography". American Journal of Sociology.100 (4), pp. 859-903

BARRETO, Miguel Ángel (2010). "El concepto de hábitat digno como meta de una política integral de áreas urbanas deficitarias críticas, para la integración social desde los derechos humanos". En: Revista INVI, 25 (69). Instituto de la Vivienda, Facultad de Arquitectura y Urbanismo, Universidad de Chile. pp. 161-187 Santiago, Chile. ISSN 0718-1299.

BARRETO, Miguel Ángel; ALCALÁ, Laura; BENÍTEZ, María Andrea; FERNÁNDEZ, María Emilia; GIRÓ, Marta; PELLI, María Bernabella; ROMAGNOLI, Venettia (2014). "Áreas urbanas deficitarias críticas como unidades de interpretación y abordaje de los nuevos territorios periurbanos". En: Karol, J.; Aón, L.; Martini, I.; Pistola, J.; Salas Giorgio, R. (Comp.) Conducir las transformaciones urbanas. Un debate sobre direcciones, orientaciones, estrategias y políticas que modelan la ciudad futura. 1. ${ }^{a}$ edición. Universidad Nacional de La Plata, 2014. Tomo II. E-Book ISBN 978-950-34-1133-9 - CDD 307.12. pp. 1211-1223. La Plata, Argentina.

BOND, Stephanie y PARKER, W. (2000). "The components of density and the dimensions of residential segregation" Population Research and Population Policy, 6 (19).

BOURDIEU, Pierre. (2001). "Los tres estados del capital". En: Sociológica, UAM-Azcapotzalco (5), pp.11-17. México.

BOURDIEU, Pierre (1996). "Espacio Social y poder simbólico". Cosas dichas. Editorial Gedisa. Barcelona, España.

CAPRÓN, Guénola y GONZÁLEZ ARELLANO, Salomón (2006). "Las escalas de la segregación y de la fragmentación urbana". Revista Trace N. ${ }^{\circ} 49$.

CEPAL/CELADE, (2002) Vulnerabilidad sociodemográfica: viejos y nuevos riesgos para comunidades, hogares y personas; síntesis y conclusiones. CEPAL/CELADE, Santiago.

GARCÍA, Rolando (2006) Sistemas complejos. Conceptos, métodos y fundamentación epistemológica de la investigación interdisciplinaria. Editorial Gedisa, Barcelona, España.

GOTMAN, Anne (1990). "Stratégies résidentielles, stratégiesde la recherche". En: Bomvalet C. et Fribourg A.-M. (dir.), Stratégiesrésidentielles. pp. 23-34. Paris, Francia.

KAZTMAN, Rubén (2001). "Seducidos y abandonados: el aislamiento social de los pobres urbanos". En: Revista de la CEPAL (75). CEPAL, diciembre. pp. 171-189 Santiago, Chile.

MAYORGA HENAO, José (2010) Planeación de equipamientos colectivos. Elaboración de una política estratégica de integración social de la población en estado de pobreza. Pontificia Universidad Javeriana. Bogotá, Colombia.

MERA, Gabriela. (2014). "De la localización a la movilidad: propuestas teórico-metodológicas para abordar la segregación espacial urbana". Cuaderno urbano 17 (17), Facultad de Arquitectura y Urbanismo, Universidad Nacional del Nordeste. Pp. 25-46. Resistencia, Argentina. ISSN 1666-6186.

MORÍN, Edgard (1990) Introducción al pensamiento complejo. Editorial Gedisa, Barcelona, España.

SABATINI, Francisco (2003). "La segregación social del espacio urbano en las ciudades de América Latina". Documentos del Instituto de Estudios Urbanos, Serie Azul, 35. Editorial Pontificia Universidad Católica. Santiago de Chile, Chile.

SALAZAR, Sandra; DEVOTO MAGOFKE, Carolina (2010). "Variables de sustentabilidad para la construcción de paisaje habitacional" Cuadernos de vivienda y urbanismo. ISSSN 2145-0226. 3 (6), juliodiciembre de 2010. pp. 198-219. Bogotá, Colombia.

SEGURA, Ramiro (2010). "Cartografías discrepantes. La ciudad de la Plata vista y vivida desde la periferia". Revista Periferia, 1 (2).

SEGURA, Ramiro (2012). "Elementos para una crítica de la noción de segregación residencial socio-económica: desigualdades, desplazamientos, e interacciones en la periferia de La Plata". Quid 2 (16).

WHITE, Michael (1983). "The Measurement of Spatial Segregation". The American Journal of Sociology, 5 (88). 